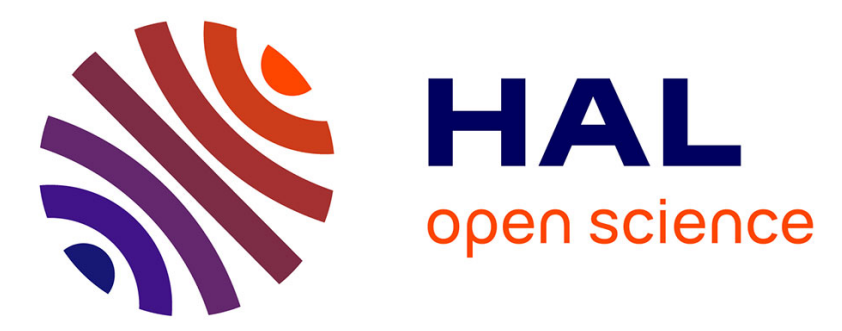

\title{
Rate equation reformulation including coherent excitation: application to periodic protocols based on spectral hole-burning
}

Yoann Attal, Perrine Berger, Loïc Morvan, Pascale Nouchi, Daniel Dolfi, Thierry Chanelière, Anne Louchet-Chauvet

\section{To cite this version:}

Yoann Attal, Perrine Berger, Loïc Morvan, Pascale Nouchi, Daniel Dolfi, et al.. Rate equation reformulation including coherent excitation: application to periodic protocols based on spectral hole-burning. Journal of the Optical Society of America B, 2018, 35 (6), pp.1260. 10.1364/JOSAB.35.001260 . hal-02106653

\section{HAL Id: hal-02106653 \\ https://hal.science/hal-02106653}

Submitted on 9 May 2019

HAL is a multi-disciplinary open access archive for the deposit and dissemination of scientific research documents, whether they are published or not. The documents may come from teaching and research institutions in France or abroad, or from public or private research centers.
L'archive ouverte pluridisciplinaire $\mathbf{H A L}$, est destinée au dépôt et à la diffusion de documents scientifiques de niveau recherche, publiés ou non, émanant des établissements d'enseignement et de recherche français ou étrangers, des laboratoires publics ou privés. 


\title{
Rate equation reformulation including coherent excitation: application to periodic protocols based on spectral hole-burning
}

\author{
Yoann Attal ${ }^{1,2,3}$, Perrine Berger ${ }^{1}$, Loïc Morvan ${ }^{1}$, Pascale Nouchi ${ }^{1}$, Daniel Dolfi ${ }^{1}$, Thierry \\ Chanelière ${ }^{3}$, And Anne Louchet-Chauvet ${ }^{3, *}$ \\ ${ }^{1}$ Thales Research and Technology, 1 av. Augustin Fresnel, 91767 Palaiseau, France \\ ${ }^{2}$ Thales Communication and Security SAS, 92230 Gennevilliers, France \\ ${ }^{3}$ Laboratoire Aimé Cotton, CNRS UMR9188, Univ. Paris-Sud, ENS Paris-Saclay, Université Paris-Saclay, bâtiment 505, 91405 Orsay, FRANCE \\ *Corresponding author: anne.chauvet@u-psud.fr

A large number of signal processing protocols are based on recording a spectral pattern via spectral holeburning in an inhomogeneously broadened absorption profile. We present a simulation method specifically designed for periodic excitation sequences leading to the creation of a spectral pattern. This method is applicable to any multilevel atomic structure. The atomic variables' coherent dynamics is solved for a single temporal excitation step. The result is expressed as an equivalent population transfer rate. This way, the whole sequence is described as a matrix product and the steadystate of the system under periodic excitation is easily derived. The propagation through the atomic medium is fully decoupled from the temporal evolution. We apply this method to the engraving of a spectral grating in a large absorption Tm:YAG sample for wideband spectral analysis. (c) 2018 Optical Society of America

OCIS codes: (020.1670) Coherent optical effects; (160.5690) Rare-earthdoped materials; (070.1170) Analog optical signal processing.

http://dx.doi.org/10.1364/ao.XX.XXXXXX

\section{INTRODUCTION}

Because of the spectroscopic properties of rare earth ion-doped crystals, a wide range of applications have been proposed in these systems. Indeed, at low temperatures (typically $4 \mathrm{~K}$ ), coherence and population lifetimes of rare earth ions can reach tens to hundreds of microseconds and from milliseconds to seconds, respectively. Besides, the inhomogeneous linewidth of the absorption profile can be as broad as a few GHz. Using these properties, complex spectral patterns can be imprinted on the absorption spectrum via spectral hole-burning. Early experiments performed in the 80's involved pulsed lasers [1-4] and validated the principle of spectro-temporal processing using organic molecules embedded in solids. The adequacy between the development of optoelectronic components and the properties of rare earth ion-doped materials fostered at the same time new proposals based on frequency-swept laser for example [5-7] and high-performance experimental demonstrations in crystalline inorganic solids [8-10]. Applications emerge in the field of optically carried radio-frequency signal processing leading to a significant improvement of the readiness level [11, 12]. More recently, using the same tools pushed at a higher spectral resolution, many perspectives have been considered for quantum information processing and primarily quantum memories [13]. In both cases, classical or quantum, the implementation and the modeling of the programmed pattern is critical to evaluate the overall processing efficiency.

The spectral patterns can be periodic spectral gratings as in quantum memories based on the atomic frequency comb protocol [14] or chirped spectral gratings as in time-to-frequency Fourier transform [15] and time-reversal architectures [16]. They can be a juxtaposition of several periodic gratings with different spacings, allowing for a configurable manipulation of optical pulses including compression and stretching operations [17]. They can be spectro-spatial gratings as in the so-called "rainbow" spectral analyzer [18] or in true-time delay generators [19]. More simply, the spectral pattern can be one spectral hole as in ultrasound-optical tomography-related architectures [20], quantum memories based on slow light [21], or laser stabilization architectures $[22,23]$.

The performance of all these architectures is directly linked to the exact shape of the spectral pattern programmed in the absorption profile. Therefore, an accurate representation of the light-atom interaction during the programming step is necessary. Besides, a large grating contrast or hole depth cannot be obtained without accumulation due to the generally low optical pumping efficiency [24]. In that case, coherence and population relaxation must be first taken into account. Additionally, a narrow band laser usually resolves the rare-earth hyperfine structure which may be due to the nuclear-electric-quadrupole and/or Zeeman interactions. The population redistribution between the different subslevels cannot be neglected and has to be included explicitly in the model. This point is critical $[25,26]$ and motivates us to propose an accurate description including the hyperfine states 


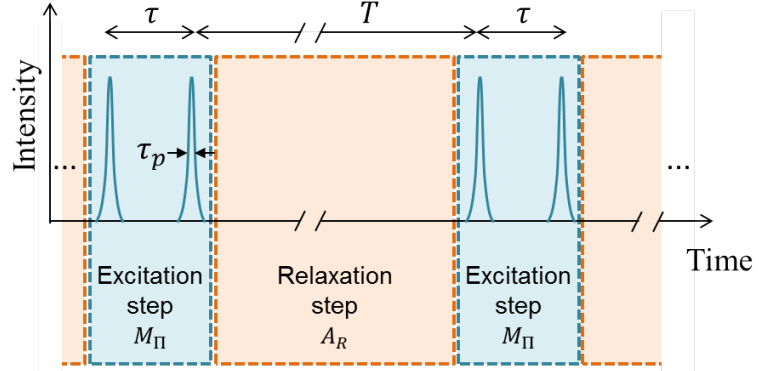

Fig. 1. Chronogram of a periodic sequence of pulse pairs as considered in [27] for example. $T$ and $\tau$ are the inter- and intraspacing of the pairs, and $\tau_{p}$ is the duration of one pulse. $M_{\Pi}$ and $A_{R}$ refer to the excitation and relaxation matrices which will be introduced in the present paper.

(under magnetic field for Tm:YAG). Finally, a large absorption, meaning an optically thick medium is generally required to provide large diffraction efficiency. This latter defines as the intensity of the detected echo with respect to the incoming signal scales the performance of the architecture. Therefore, in the purpose of determining the technical and physical limitations of a given architecture, an adequate model should therefore account for coherent evolution, population accumulation, and field propagation.

Many simulations of accumulated photon echoes or accumulated spectral hole-burning were published over the past 15 years, stimulated by the new architectures [26-30]. All of them deal with a repetitive sequence of pulse pairs interleaved with a free evolution step (see Fig. 1 for notations). Although the free evolution step is always accurately described by a transfer matrix [27], the analytic modeling of the excitation systematically requires hypotheses that potentially limit the case studies. In [27] the excitation pulses are assumed to be weak, square, and short: $\tau_{p} \ll \tau$ and $\tau_{p} \ll 1 / \Gamma_{i n h}$ where $\Gamma_{i n h}$ is the inhomogeneous broadening of the optical transition. In [28] the pulses are assumed shorter than all the relaxation times including the optical coherence lifetime of the excited transition $T_{2}$ and $1 / \Gamma_{i n h}$. In [29], the material is assumed optically thin (low absorption) and the excited level empty at the beginning of each new cycle. In [30], the authors consider an optically thin medium, square pulses, and neglect the coherence decay within each pulse pair $\left(\tau \ll T_{2}\right)$. Finally, in [26], a weak excitation is assumed and the coherence decay within each excitation is neglected. In all these papers [26-30], the coherence is assumed to be lost after one cycle: $T_{R} \gg T_{2}$.

In [31], DeVries et al. numerically solve the optical Bloch equations in a two-level system within one temporal excitation step, ie a pair of pulses, and translate the action of this pulse as a rate equation onto the population difference. This is justified if $T \gg T_{2}$. The excitation is therefore modeled via a matrix product, just like relaxation, which makes it possible to derive the steady state of the system after a large number of periods. Still, they assume that $\tau \ll T_{1} \ll T$, where $T_{1}$ is the population lifetime of the excited state.

In this paper, we propose an extension of the simulation method developed in [31]. The method we propose is applicable to the protocols based on periodic excitation, whatever the temporal excitation step (including single pulse, pairs of pulses, chirped pulses, etc). We generalize our approach to a multilevel atomic structure, with no restriction in terms of level number, relaxation paths, or number of optically excited transitions, and with no limitation on the excitation power. We assume that all the atomic coherences are lost after one cycle $\left(T \gg T_{2}\right)$ but make no other assumption on population lifetimes, coherence lifetimes and the period of the excitation. The full evolution of the system under periodic excitation is expressed as a matrix product and the steady-state is derived as the limit of a geometric sequence. The propagation of the excitation in the volume of the atomic medium is performed separately.

This approach is frugal in terms of computational power. The coherent excitation is solved numerically only once and is then only expressed as a population transfer matrix. The global evolution is solely modeled by matrix products which can be implemented efficiently using standard linear algebra libraries.

This method is applicable to any protocol involving a periodic sequence of spectral hole-burning which leads to a steady state of the atomic population distribution. Therefore it is relevant to all of the programmable filters cited above [14-23], provided the filter is periodically refreshed.

Despite a clear motivation to model the hole-burning dynamics in rare earth ion-doped crystals for optical signal processing, our model could certainly be used in other domains where the interplay between coherent evolution and accumulation appear under pulsed excitation. This is the case for multilevel atomic vapors under femtosecond pulses where the internal dynamics is driven by the accumulation of many pulses described by an abundant bibliography [32-39] finding application in optical metrology, or for complex molecules and semiconductor structures whose fast response can be studied by coherent spectroscopy [40]. We first present our method and give the explicit formulation of the steady state in the general case. As a testbed, we apply it to a two-level atom under a periodic excitation made of Gaussian pulse pairs whose dynamics can be partially derived analytically. Then we apply our method to the creation of a steady-state wideband spectral grating in a Tm:YAG crystal under magnetic field. We also present in the appendix a general, practical formulation of Bloch vector equations that will help to apply this method to a general atomic structure with an arbitrary number of levels and transitions.

\section{GENERAL DESCRIPTION OF THE METHOD}

We consider a $N_{L}$-level atomic system excited with a $T$-periodic pulse sequence driving one or more atomic transitions. Each cycle is composed of an excitation step followed by a free evolution step. The atomic levels are written $|i\rangle_{i=1 \ldots N_{L}}$.

\section{A. Matrix formulation of the excitation}

The effect of one excitation step on the atomic populations $n_{i}\left(i=1 \ldots N_{L}\right)$ can be expressed as a set of effective dimensionless population transfer rates $\left\{\Pi_{i j}\right\}_{\left(1 \leq i, j \leq N_{L}\right)}$ such that:

$$
n_{i}^{(+)}-n_{i}^{(-)}=-\sum_{j} \frac{\Pi_{i j}}{2}\left(n_{i}^{(-)}-n_{j}^{(-)}\right),
$$

where ${ }^{(-)}$and ${ }^{(+)}$label the populations immediately before and after one excitation step respectively. With this definition, the population transfer rate lies between 0 (no change in the population) and 2 (full population inversion). The transfer rates $\Pi_{i j}$ are calculated so that the optical excitation is equivalent to an instantaneous modification of the population by fully neglecting population relaxation during the excitation step. They are obtained analytically in the perturbative regime, or by numerically 
solving optical Bloch equations, as further described in Sec. E. If the excitation steps are separated by free evolution intervals where the atomic coherences are lost, then Eq. (1) is sufficient to describe any excitation step. We define the population vector $\Psi=\left(n_{1} ; n_{2} ; \ldots ; n_{N_{L}}\right)$ with $\sum_{i=1}^{N_{L}} n_{i}=1$ and $0 \leq n_{i} \leq 1$. Eq. (1) translates into a matrix product:

$$
\Psi_{n}^{(+)}=M_{\Pi} \Psi_{n}^{(-)},
$$

where $\Psi_{n}^{(-)}$and $\Psi_{n}^{(+)}$are the population vectors before and after the $n^{\text {th }}$ excitation, and:

$$
M_{\Pi}=I_{N_{L}}+\sum_{i} \sum_{j \neq i} \frac{\Pi_{i, j}}{2}\left(-e_{i, i}+e_{i, j}+e_{j, i}-e_{j, j}\right),
$$

$e_{i, j}$ are square matrices of size $N_{L}$ whose element $(i, j)$ is one and all the others are zero. $I_{N_{L}}$ stands for the identity matrix of size $N_{L}$.

\section{B. Free evolution}

The free evolution is described with a linear differential system with constant coefficients acting on the populations as relaxation process: $\partial_{t} \Psi=K \Psi$ where the coefficients of the $N_{L} \times N_{L}$ matrix $K$ involve the population relaxation rates. The solution to this differential system is given by the matrix exponential $A_{R}=e^{T_{R} K}$ where $T_{R}$ is the free evolution duration, so that:

$$
\Psi_{n+1}^{(-)}=A_{R} \Psi_{n}^{(+)} .
$$

\section{System evolution and steady state}

Both steps of the periodic sequence are now written as matrix products. This way, the temporal evolution over a large number of cycles is accessible with very limited computational resources involving standard algebra routines. The populations before the $n^{\text {th }}$ excitation are given by a geometric sequence:

$$
\Psi_{n}^{(-)}=\left(A_{R} M_{\Pi}\right)^{n} \Psi_{0}^{(-)} .
$$

Since both matrices $M_{\Pi}$ and $A_{R}$ operate within the subensemble of population vectors, we easily show that these matrices are stochastic, ie the sum of the elements in each column equals 1. Therefore their product $A_{R} M_{\Pi}$ is a stochastic matrix as well, and its eigenvalues are either 1 or within the open unit disk. Therefore the sequence $\left\{\Psi_{n}^{(-)}\right\}_{n \in \mathbb{N}}$ converges towards a steady state $\Psi_{\infty}^{(-)}$, which is an eigenvector of $A_{R} M_{\Pi}$ with eigenvalue 1. Similarly, one may define the steady state $\Psi_{\infty}^{(+)}$as the limit of the geometric sequence $\left\{\Psi_{n}^{(+)}\right\}_{n \in \mathbb{N}}$.

This steady state can be derived for any atom in the absorption profile, taking into account the detuning between the atomic resonance and the excitation frequency when determining the population transfer rates $\Pi_{i j}$. The atomic populations at frequency $v$ oscillate between $\Psi_{\infty}^{(-)}(v)$ and $\Psi_{\infty}^{(+)}(v)$ with period $T$. Therefore the shape of the spectral pattern imprinted in the absorption spectrum oscillates at the excitation repetition rate. In the prospect of classical or quantum information processing, we are primarily interested in stable profiles. This implies that the repetition rate of the excitation must be high enough to avoid significant population relaxation between two successive excitations. We point out that the atomic state at any intermediate time between excitations can be predicted if necessary, using $\Psi_{\infty}^{(+)}(v)$.

\section{Propagation in an optically thick medium}

A scalar electric field $\mathcal{E}(t, \vec{r})$ whose central frequency is $v_{0}$ is described by its slowly time-varying amplitude $E(t, \vec{r})$ such that $\mathcal{E}(t, \vec{r})=E(t, \vec{r}) e^{-2 i \pi v_{0} t}+c . c$. . Alternatively, it is described by $\tilde{E}(\nu, \vec{r})$ as the temporal Fourier transform of $E(t, \vec{r})$. In dielectric medium, the Maxwell wave propagation equation for the frequency domain function $\tilde{E}(v, \vec{r})$ reads as (see for example [41, 42]):

$$
\Delta \tilde{E}(\nu, \vec{r})+k^{2}\left(1-\frac{i}{k}(1+i \mathcal{H}) \alpha(v, \vec{r})\right) \tilde{E}(\nu, \vec{r})=0,
$$

where $k$ is the length of the wavevector $\vec{k}$ and $\alpha(v, \vec{r})$ is the absorption coefficient of the medium at position $\vec{r}$ and frequency $v$. The term $(1+i \mathcal{H}) \alpha(v, \vec{r})$ represents the medium susceptibility. This latter directly includes the causality (Kramers-Kronig relations $[42,43])$ where $\mathcal{H}$ stands for the Hilbert transform [3].

We consider the excitation field propagating along $\hat{z}$. This will be explained in the following paragraph. It is now possible to define optically thin slices in the atomic medium orthogonal to $\hat{z}$ and use the input field $\tilde{E}(v, z=0)$ to calculate the steady-state populations in the first slice. The mean value of the populations $\frac{\Psi_{\infty}^{(-)}+\Psi_{\infty}^{(+)}}{2}$ provides the absorption coefficient $\alpha(v, z=0)$ to be inserted in Eq. (6), which in turn yields the field that excites the second slice. This procedure can be repeated to obtain the absorption coefficient $\alpha(v, z)$ over the whole depth of the crystal. We will show in section C.3 how to avoid a new numerical resolution of the optical Bloch equations at each iteration.

We now identify the conditions for which the propagation axis $\hat{z}$ is well defined. The atomic medium can be illuminated by one laser beam or more, but all beams must enter the material from the same face and there must exist a set of orthogonal spatial coordinates $(x, y, z)$ such that the slowly time-varying envelope $E(t, \vec{r})$ can be written as:

$$
E(t, \vec{r})=E_{0}(x, y) \cdot f(z, t) .
$$

With only one laser beam, Eq. (7) is directly satisfied when the $\hat{z}$ is the propagation axis (see Fig. 2a and d). With several beams, the angles between the beams must satisfy $\theta \ll w / L$ (where $w$ is the smallest beam waist in the medium and $L$ is the medium thickness along the $\hat{z}$ axis) in order to fulfill Eq. (7) (see Fig. $2 b$ and e).
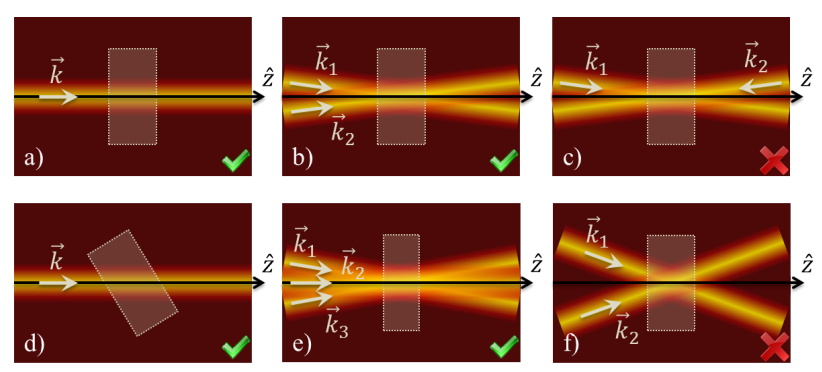

Fig. 2. Beam arrangements on the crystal. The beams are represented in yellow. The crystal is drawn as a dotted rectangle, and the propagation axis $\hat{z}$ as a horizontal arrow. The configurations a), b), d) and e) fulfill Eq. (7) whereas the configurations c) and f) do not. 


\section{E. Calculating the population transfer rate}

Let $\tilde{\Omega}_{i j}(v)$ be the Fourier transform of the time-dependent Rabi frequency relative to the transition $|i\rangle \rightarrow|j\rangle$. In the case of weak field excitation $\left(\left|\tilde{\Omega}_{i j}(v)\right|^{2} \ll 1\right)$ and ignoring population relaxation during the excitation, the population transfer rate is given by the excitation power spectrum, convoluted by the atomic response:

$$
\Pi_{i j}(v)=2\left|\tilde{\Omega}_{i j}(v)\right|^{2} \otimes \mathcal{L}_{\Gamma_{h}}(v),
$$

where $\mathcal{L}_{\Gamma_{h}}(v)$ is the normalized Lorentzian function whose FWHM is the homogeneous linewidth of the transition $\Gamma_{h}=$ $1 /\left(\pi T_{2}\right)$, and verifying $\int_{\mathbb{R}} \mathcal{L}_{\Gamma_{h}}(v) d v=1$. Equation (8) is deduced from a second order expansion of the optical Bloch equations and has been derived in the early days of spectro-temporal processing with hole-burning materials [5].

When coherence and population relaxations cannot be neglected, under strong excitation [44], a numerical integration is necessary. We use the Bloch vector formalism, so as to manipulate only real variables. The transfer matrix $M_{\Pi}$ is then real. When the system is composed of more than 2 levels, the Bloch vector equations get more complex [45]. In the appendix of this paper, we give a practical method to write the Bloch vector dynamics in an arbitrary level structure.

\section{SIMPLE EXAMPLE : OPTICALLY THIN TWO-LEVEL SYSTEM}

In this section we explain our simulation method with a simple comparative example for which the analytic expression of the transfer rate is partially valid. We consider a two-level system excited with a periodic pulse sequence. Each period of this sequence is composed of a temporal excitation step (a pair of monochromatic Gaussian pulses separated by $\tau$ ) and a $T_{R}$-long free evolution.

\section{A. Optical Bloch equations}

The two levels are labeled $|g\rangle$ and $|e\rangle$. The transition frequency is noted $v$. The atomic populations verify $n_{g}+n_{e}=1$. From the density matrix $\left(\rho_{i j}\right)_{i, j \in\{g, e\}}$ we define the slowly varying quantities:

$$
\left\{\begin{aligned}
\sigma_{e g} & =\rho_{e g} e^{2 i \pi v_{0} t} \\
\sigma_{g e} & =\rho_{g e} e^{-2 i \pi v_{0} t} \\
\sigma_{e e} & =\rho_{e e}=n_{e} \\
\sigma_{g g} & =\rho_{g g}=n_{g}
\end{aligned}\right.
$$

where $v_{0}$ is the excitation frequency. The Bloch vector is defined as $\vec{B}=(U ; V ; W)$ where $U$ and $V$ represent the complex coherence of the optical transition such that $U=\sigma_{g e}+\sigma_{g e}$, $V=i\left(\sigma_{e g}-\sigma_{g e}\right)$, and $W=\sigma_{e e}-\sigma_{g g}$ is the population difference between the two levels. The optical Bloch equations now read as:

$$
\partial_{t} \vec{B}=\vec{\beta} \times \vec{B}+\vec{R},
$$

where $\times$ is the vector product. The control vector is defined as: $\vec{\beta}=\left(\Re(\Omega(t)) ; \Im(\Omega(t)) ;-2 \pi\left(v_{0}-v\right)\right)$ where $\Omega(t)$ is the slowly varying complex Rabi frequency (in rad.s ${ }^{-1}$ ). The relaxation vector contains the population and coherence lifetimes $T_{1}$ and $T_{2}$ :

$$
\vec{R}=\left(-\frac{U}{T_{2}} ;-\frac{V}{T_{2}} ;-\frac{W+1}{T_{1}}\right) .
$$
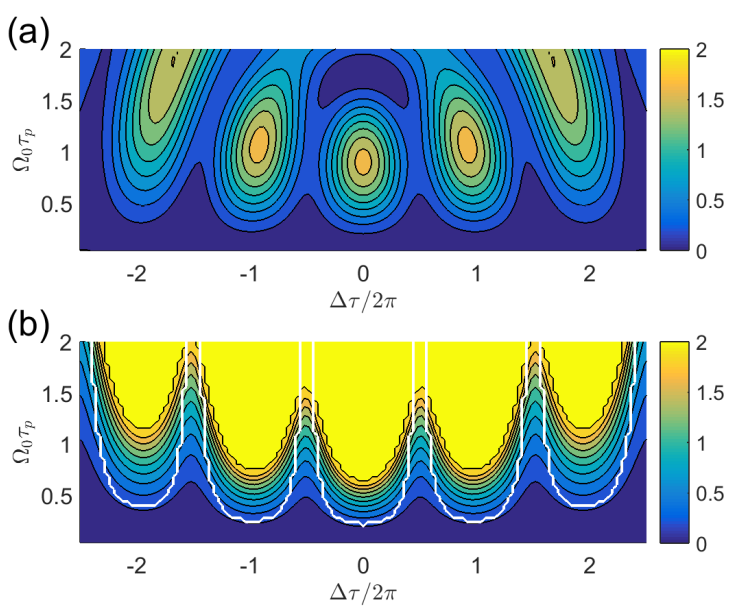

Fig. 3. Population transfer rate $\Pi_{g e}$ in the case of a two-level system excited with a pair of Gaussian pulses with $\tau=4 \mu \mathrm{s}$, $\tau_{p}=0.5 \mu$ s and $T_{2}=40 \mu \mathrm{s}$. The simulation uses the full optical Bloch equations (a) or the weak field approximation (b). The white line represents the boundary $|\tilde{\Omega}(v)|^{2} \geq 0.5$, ie values of $\Omega_{0}$ and $v$ where the weak field approximation is invalid.

\section{B. Derivation of the effective population transfer rate based on the optical Bloch equation}

We numerically solve the Bloch vector equation (10) over one excitation, starting from a pure population state (ie $U=V=$ $0)$. Because, we neglect the population relaxation during the excitation step, the pumping rate $\Pi_{g e}$ doesn't depend on the initial population $W^{(-)}$. As a simple example, if the excitation is a $\pi$-pulse, then $\Pi_{g e}=2$ and we find $W^{(+)}=-W^{(-)}$. In other words, an atom in the ground state $W^{(-)}=-1$ goes to the excited state $W^{(+)}=1$ and vice versa (if $W^{(-)}=1$ then $\left.W^{(+)}=-1\right)$. Any intermediate value of $W^{(-)}$between -1 et 1 would be inverted and verifies $W^{(+)}=-W^{(-)}$. The fact that the pumping rate $\Pi_{g e}$ doesn't depend on the initial population ensures the converges of the matrix product toward the steadystate as described in C.

For an excitation step composed of a pair of monochromatic Gaussian pulses, we introduce the effective population transfer rate $\Pi_{g e}(v)$ as defined in Eq. (1). $\Pi_{g e}$ depends on the detuning $\Delta=2 \pi\left(v_{0}-v\right)$ and on the maximum Rabi frequency $\Omega_{0}$. It is deduced from the simulation by: $\Pi_{g e}=1-\left(W^{(+)} / W^{(-)}\right)$. We compare these population transfer rates with the ones obtained from the weak field approximation using Eq. (8). The results of the simulation are shown in Fig. 3. We confirm that under the weak field condition, the population transfer rate is close to the excitation power spectrum, as expected for a Gaussian envelope modulated by $1 / \tau$ for $\left.\tilde{\Omega}(v)\right|^{2}$. This is expected when $T_{2} \gg \tau$. However, the weak field approximation is restricted to a very small range of excitation parameters, since it significantly deviates from the complete solution as soon as $|\tilde{\Omega}(v)|^{2} \geq 0.5$. For strong pulses, the modulation is still present. The population dynamics is more complex and can be seen as generalized Ramsey fringes (usually restricted to $\pi / 2$-pulse pair in textbook).

Once the transfer rate $\Pi_{g e}(v)$ is derived (analytically or numerically), one writes the excitation and relaxation matrices $M_{\Pi}$ 


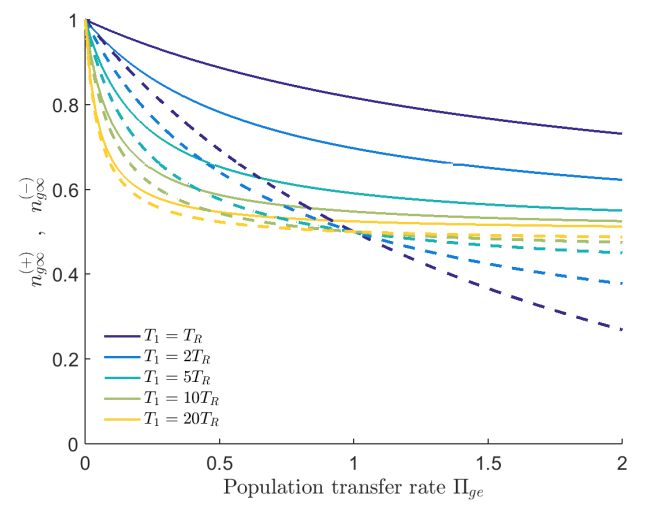

Fig. 4. Steady-state ground state population $n_{g, \infty}$ in a twolevel system excited with a periodic pulse sequence whose excitation is translated as a population transfer rate $\Pi_{g e}$. The solid and dashed lines represent $n_{g, \infty}^{(-)}$and $n_{g, \infty}^{(+)}$before and after the excitation, respectively. Various values of the ratio $T_{1} / T$ are explored, keeping $T_{1} / T_{2}=150$.

and $A_{R}$ for each frequency $v$ according to Eq. (3):

$$
\begin{aligned}
& M_{\Pi}=\left(\begin{array}{cc}
1-\Pi_{g e} / 2 & \Pi_{g e} / 2 \\
\Pi_{g e} / 2 & 1-\Pi_{g e} / 2
\end{array}\right), \\
& A_{R}=\exp \left[T\left(\begin{array}{cc}
0 & 1 / T_{1} \\
0 & -1 / T_{1}
\end{array}\right)\right] .
\end{aligned}
$$

The population after $n$ cycles of excitation and free evolution is given by Eq. (5). The steady-state population $\Psi_{\infty}^{(-)}$is the eigenvector of $A_{R} M_{\Pi}$ with eigenvalue 1 , defined as $\Psi_{\infty}^{(+)}=$ $M_{\Pi} \Psi_{\infty}^{(-)}$. In Fig. 4 we show the steady-state populations in the ground state $n_{g, \infty}^{(-)}$and $n_{g, \infty}^{(+)}$for various population lifetimes as a function of the transfer rate $\Pi_{g e}$. As expected, the two steady states are closer to each other when $T_{1} / T$ is large.

\section{CASE OF A WIDEBAND SPECTRAL GRATING IN OP- TICALLY THICK TM:YAG}

In this section we consider a programming sequence composed of a pair of parallel linear chirps, in a $\mathrm{Tm}^{3+}$ :YAG crystal under magnetic field. This configuration is close to the situation of the "rainbow" RF spectrum analyzer, where a wideband spectrospatial grating is burnt via a periodic sequence of frequency chirped and angularly scanned beams $[18,46]$. Here we simplify the excitation sequence by considering collinear programming beams, in order to be able to study the gratings in the sole frequency domain. We first describe the programming pulse sequence, then specify the energy level structure and relaxation mechanisms in Tm:YAG, and finally apply our simulation method.

\section{A. Excitation sequence}

We consider a periodic pulse sequence that creates a wideband spectral interference pattern (see Fig. 5). In one period, two laser pulses are generated with duration $\Delta_{t}$ and their frequency is linearly chirped over $\Delta_{v}=20 \mathrm{GHz}$. The chirp duration $\Delta_{t}$ is varied from $350 \mu$ s to $40 \mathrm{~ms}$. The chirp rate is defined as $r=\Delta_{v} / \Delta_{t}$. The two chirped pulses partially overlap, with a

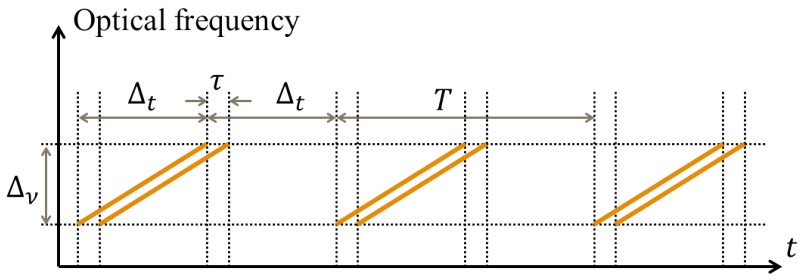

Fig. 5. Chronogram for creating an accumulated wideband spectral grating.

time separation $\tau=100 \mathrm{~ns}$. The corresponding time varying phases can be written as

$$
\varphi_{1}(t)=2 \pi\left(v_{0} t+\frac{\Delta_{v}}{2 \Delta_{t}}(t+\tau / 2)^{2}\right)
$$

and

$$
\varphi_{2}(t)=2 \pi\left(v_{0} t+\frac{\Delta_{v}}{2 \Delta_{t}}(t-\tau / 2)^{2}\right),
$$

giving linear frequency sweeps of the instantaneous frequencies $\partial_{t} \varphi_{1}(t)=2 \pi\left(v_{0}+\frac{\Delta_{v}}{\Delta_{t}}(t+\tau / 2)\right)$ and $\partial_{t} \varphi_{2}(t)=$ $2 \pi\left(v_{0}+\frac{\Delta_{v}}{\Delta_{t}}(t-\tau / 2)\right)$.

The repetition rate is set such that $T=2 \Delta_{t}$. Therefore the incident excitation field restricted to $t \in\left[-\Delta_{t} / 2+\tau / 2, \Delta_{t} / 2-\right.$ $\tau / 2$ ] (see Fig. 5) reads as:

$$
E(x, y, z, t)=E_{0}(x, y) e^{-i k z} \cdot\left[e^{i \varphi_{1}(t)}+e^{i \varphi_{2}(t)}\right]
$$

In the following, the transverse shape of the excitation will be neglected: $E_{0}(x, y)=E_{0}$.

We focus on the atoms at least $100 \mathrm{MHz}$ from the edges of the chirp span. This allows us to restrict the effective excitation frequency span and duration to $v+[-100 \mathrm{MHz}, 100 \mathrm{MHz}]$ and $200 \mathrm{MHz} / r$, respectively. This way, the effective excitation duration is only $1 \%$ of the total excitation sequence period. Therefore, the rest of the sequence period can be regarded as a free evolution step with duration $T_{R} \simeq T \gg T_{2}$, so that our simulation method is applicable.

\section{B. Level structure and relaxation paths}

We focus on the optical transition at $793 \mathrm{~nm}$ in Tm:YAG. The relevant energy levels are shown in Fig. 6. Without magnetic field, the system is composed of three levels ${ }^{3} \mathrm{H}_{6},{ }^{3} \mathrm{H}_{4}$ and ${ }^{3} \mathrm{~F}_{4}$ that we name ground $|g\rangle$, excited $|e\rangle$, and metastable $|m\rangle$, respectively. An external magnetic field splits each electronic level into two nuclear spin sublevels with frequency splittings $\Delta_{g}$ and $\Delta_{e}$ in the ground and excited states, respectively [47].

We assume the light polarization and magnetic field to be parallel to the [111] axis of the crystal. Among the six differently oriented $\mathrm{Tm}^{3+}$ sites in YAG, as defined in [48], the so-called sites 2,4 and 6 do not participate in the light-atom interaction because the transition dipole moment is orthogonal to the electric field. For the magnetically and optically equivalent sites 1, 3, and 5, the optical transitions that connect two levels of opposite nuclear spin (namely $\left|g_{1}\right\rangle \rightarrow\left|e_{2}\right\rangle$ and $\left|g_{2}\right\rangle \rightarrow\left|e_{1}\right\rangle$ ) are forbidden so we consider only two separate optical transitions $\left(\left|g_{1}\right\rangle \rightarrow\left|e_{1}\right\rangle\right.$ and $\left.\left|g_{2}\right\rangle \rightarrow\left|e_{2}\right\rangle\right)$ [47].

The nuclear spin is mostly preserved during the relaxation process. Only $2 \%$ of the atoms in one of the excited states decay directly to the ground state sublevel with the same nuclear spin 


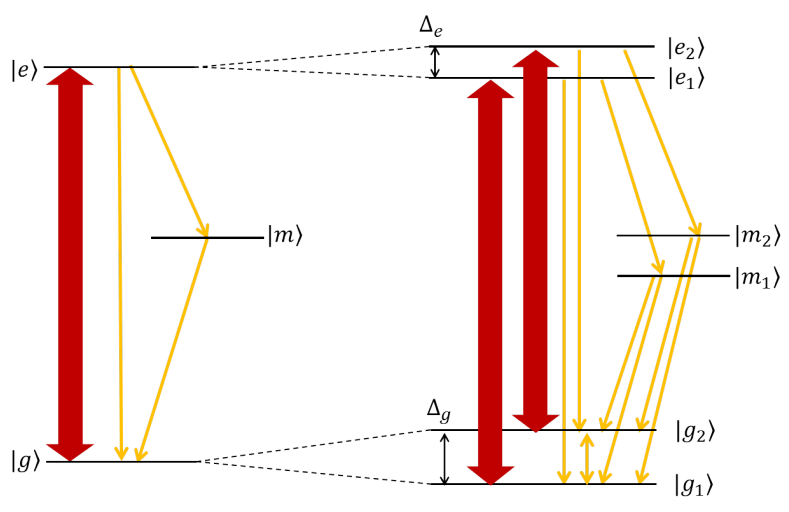

Fig. 6. Atomic structure of $\mathrm{Tm}^{3+}$ ions without (left) and with external magnetic field (right). Thick and thin arrows represent the optical transitions of interest and the relaxation paths, respectively.

(e.g. from $\left|e_{1}\right\rangle$ to $\left|g_{1}\right\rangle$ ). The remaining $98 \%$ also decay to the ground states but via the metastable state, with a $33 \%$ chance to reach a ground state sublevel with a different nuclear spin. The branching parameters mentioned here were measured experimentally in a $0.5 \% \mathrm{Tm}$ :YAG crystal. The population lifetimes are $500 \mu \mathrm{s}$ for the excited state and $15 \mathrm{~ms}$ for the metastable state at $3 \mathrm{~K}$. The optical coherence lifetime along the two optical transitions is $T_{2}=20 \mu \mathrm{s}$.

The presence of a magnetic field significantly increases the hole-burning lifetime. In our configuration, the relaxation process between the ground Zeeman sublevels has a lifetime of $26 \mathrm{~s}$ at $3 \mathrm{~K}$. Additionally, the Zeeman structure is now well-resolved by the laser such as the redistribution between the different sublevels has be considered to fully model the imprinted pattern. We adjust the magnetic field to have a ground state splitting equal to half of the periodic pattern period. This so-called Interlaced Spin Grating configuration maximizes the accumulated grating contrast as demonstrated in [26]: $2 \Delta_{g e} \tau=1$ where $\Delta_{g e}=\Delta_{g}-\Delta_{e}$. This corresponds to a $127 \mathrm{G}$ magnetic field along the [111] axis. Finally, we consider a total optical depth $\alpha_{0} L=2$.

\section{Simulation}

\section{C.1. Bloch vector equations}

Our system contains 6 atomic levels and 2 optical transitions $\left|g_{1}\right\rangle \rightarrow\left|e_{1}\right\rangle$ and $\left|g_{2}\right\rangle \rightarrow\left|e_{2}\right\rangle$. We define two Bloch vectors $\vec{B}_{1}=\left(U_{1} ; V_{1} ; W_{1}\right)$ and $\vec{B}_{2}=\left(U_{2} ; V_{2} ; W_{2}\right)$ corresponding to the two optically excited transitions. Since the 2 two-level systems are not independent due to relaxation paths, we also include the populations in states $\left|g_{1}\right\rangle$ and $\left|g_{2}\right\rangle$ as additional variables. Finally, we include the two metastable state populations to form the 10-component vector describing our 6-level system:

$$
\vec{B}=\left(U_{1} ; V_{1} ; W_{1} ; U_{2} ; V_{2} ; W_{2} ; n_{g_{1}} ; n_{g_{2}} ; n_{m_{1}} ; n_{m_{2}}\right) \text {. }
$$

The control vectors $\vec{\beta}_{g_{1}, e_{1}}$ and $\vec{\beta}_{g_{2}, e_{2}}$ act on the Bloch vectors $\vec{B}_{1}$ and $\vec{B}_{2}$, respectively. The optical frequencies of the transitions $\left|g_{1}\right\rangle \rightarrow\left|e_{1}\right\rangle$ and $\left|g_{2}\right\rangle \rightarrow\left|e_{2}\right\rangle$ are $v_{T m}$ and $v_{T m}-\Delta_{g e}$, respectively. In the following, when there is no ambiguity, we replace couples of indices $\left(g_{i}, e_{i}\right)$ with $i$. The total incoming excitation field reads as a sum of two chirps [see Eq. (14)]. Taken separately, each of these chirps leads to a Rabi frequency $\Omega_{0}$. By using the rotating wave approximation, for the two chirps average phase $\frac{\varphi_{1}(t)+\varphi_{2}(t)}{2}$ the control vectors for the two optical transitions read as:

$$
\begin{aligned}
& \vec{\beta}_{1}=\left(\begin{array}{c}
2 \Omega_{0} \cos \pi r \tau t \\
0 \\
-2 \pi\left(r t+v_{0}-v_{T m}\right)
\end{array}\right) \\
& \vec{\beta}_{2}=\left(\begin{array}{c}
2 \Omega_{0} \cos \pi r \tau t \\
0 \\
-2 \pi\left(r t+v_{0}-v_{T m}+\Delta_{g e}\right)
\end{array}\right)
\end{aligned}
$$

The Bloch vector equation during the excitation pulse reads as:

$$
\partial_{t} \vec{B}=\vec{P}+\vec{R}
$$

where $\vec{P}$ contains the vector products:

$$
\vec{P}=\left(\begin{array}{c}
\vec{\beta}_{1} \times \vec{B}_{1} \\
\vec{\beta}_{2} \times \vec{B}_{2} \\
\frac{\beta_{1}^{(1)} V_{1}-\beta_{1}^{(2)} U_{1}}{2} \\
\frac{\beta_{2}^{(1)} V_{2}-\beta_{2}^{(2)} U_{2}}{2} \\
0 \\
0
\end{array}\right)
$$

where $\beta_{i}^{(j)}$ is the $j^{\text {th }}$ coefficient of $\vec{\beta}_{i}$. The relaxation vector $\vec{R}$ contains all the coherence and population lifetimes.

\section{C.2. Derivation of the steady-state}

The Bloch vector evolution Eq. (18) is solved numerically during the excitation step, using the built-in ode45 Matlab function, for different detunings within one spectral period $1 / \tau$ [49]. This yields the population transfer rates $\Pi_{1}$ and $\Pi_{2}$ for any particular value of $v_{T m}$ since the excitation spectrum is periodic. Again, we emphasize that this rate equation formalism includes the coherent interaction during the excitation step, provided that the free evolution steps allow the coherences to vanish completely. $\Pi_{2}$ is deduced from $\Pi_{1}$ using:

$$
\Pi_{1}(v)=\Pi_{2}\left(v+\Delta_{g e}\right) .
$$

Because of the Interlaced Spin Grating condition [26], $\Pi_{1}$ and $\Pi_{2}$ are simply shifted by $\pi$.

We now use the matrix formalism developed in Sec. 2. Let $\Psi=\left(n_{g_{1}} ; n_{e_{1}} ; n_{g_{2}} ; n_{e_{2}} ; n_{m_{1}} ; n_{m_{2}}\right)$ be the state vector of our atomic structure. The coherent evolution during the excitation step is described by the transfer matrix

$$
M_{\Pi}=\left(\begin{array}{cccccc}
1-\frac{\Pi_{1}}{2} & \frac{\Pi_{1}}{2} & 0 & 0 & 0 & 0 \\
\frac{\Pi_{1}}{2} & 1-\frac{\Pi_{1}}{2} & 0 & 0 & 0 & 0 \\
0 & 0 & 1-\frac{\Pi_{2}}{2} & \frac{\Pi_{2}}{2} & 0 & 0 \\
0 & 0 & \frac{\Pi_{2}}{2} & 1-\frac{\Pi_{2}}{2} & 0 & 0 \\
0 & 0 & 0 & 0 & 1 & 0 \\
0 & 0 & 0 & 0 & 0 & 1
\end{array}\right),
$$

whereas the $T_{R}$-long free evolution is described by the matrix $A_{R}$ obtained from a matrix exponential. With the matrices $A_{R}$ 
and $M_{\Pi}$, we determine the steady-state population vectors $\Psi_{\infty}^{(-)}$ and $\Psi_{\infty}^{(+)}$.

\section{C.3. Propagation}

The general propagation equation Eq. (6) can be significantly simplified in our case. Rigorously, Eq. (6) can lead to electric field spectrum distortion in amplitude and phase, so as a consequence the temporal shape does not correspond to the double chirp of Eq. (14) any longer. This distortion would in principle require a new numerical resolution (per slice) of the optical Bloch equations with modified control vectors $\vec{\beta}_{1}$ and $\vec{\beta}_{2}$. Nevertheless, in most engraving protocols (including the present case of double chirp) the distortions are small enough so they do not significantly change the value of the population transfer rate. One can then assume that the excitation spectrum preserves its shape such as its evolution is described by the propagation of its amplitude only (phase propagation can be neglected). This is major computational simplification.

Given the single-beam configuration in the slowly varying envelope approximation, the spectrum amplitude verifies the simplified propagation equation (see Appendix of [26] for a detailed derivation):

$$
\frac{\partial|E(v, \vec{r})|^{2}}{\partial z}+\alpha(v, \vec{r})|E(v, \vec{r})|^{2}=0 .
$$

For a periodic excitation, Eq. (22) will be solved over one period of the spectrum. To do so, we first divide the crystal into 25 optically thin slices orthogonal to the beam propagation direction $\hat{z}$. As a rule of thumb, we have found that the simulation converges satisfyingly for an optical thickness of one slice 0.1 . Depending on the total optical thickness of the medium $\alpha_{0} L$, the number of slices has to adapted accordingly. We start with a numerical calculation of the population transfer rate (over one spectral period as well). The simulation is achieved for a fixed chirp rate and a range of Rabi frequencies $\Omega_{0}$. We use the matrix formalism to calculate the average steady-state population $\left(\Psi_{\infty}^{(-)}+\Psi_{\infty}^{(+)}\right) / 2$ in the first slice of the atomic medium and derive the absorption:

$$
\alpha(v)=\alpha_{0}\left[\left(n_{g_{1}}-n_{e_{1}}\right)(v)+\left(n_{g_{2}}-n_{e_{2}}\right)\left(v+\Delta_{g e}\right)\right] .
$$

Then we use Eq. (22) to obtain the electric field that excites the second slice. We calculate the corresponding Rabi frequency over one spectral period and derive the corresponding population transfer rate by using again the values calculated initially, and interpolating them if necessary. This yields the steady-state population in the second slice. We repeat this process through the whole depth of the crystal. The distortion of the engraving field is then modeled by Eq. (22) but neglected in the control vectors dynamics that is always described as an undistorted double chirp. This later provides the population transfer rates after numerical resolution of the Bloch equations that can be tabulated once and used afterward during the propagation. As a consequence, the accuracy of our model decreases when the diffraction efficiency increases. To account for large diffraction efficiencies (few percents), one must compute the temporal shape of the electric field using Eq. (22), and numerically solve the optical Bloch equations for every slice. Depending on the desired accuracy and the available computing resources, these latter additional steps can be performed only every few slices.

\section{Results and discussion}

In the following we compare the population transfer rates obtained with the numerical resolution and the analytic result that is derived from the weak field approximation. Then we show the absorption profile throughout the whole sample depth and diffraction efficiency as computed using our matrix formalism.

\section{D.1. Population transfer rate}

We focus on atoms lying on the central bright fringe of the spectral interference pattern such that $v_{T m}=v_{0}$ in Eq.(17). The population transfer rates for a set of 8 chirp rates and a range of Rabi frequencies going from 0 to $4 \mathrm{Mrad} / \mathrm{s}$ are plotted in Fig. 7 . As expected, for weak fields, the numerical resolution of the Bloch vector equations retrieves the weak excitation limit:

$$
\Pi_{1}=\frac{2 \Omega_{0}^{2}}{r} .
$$

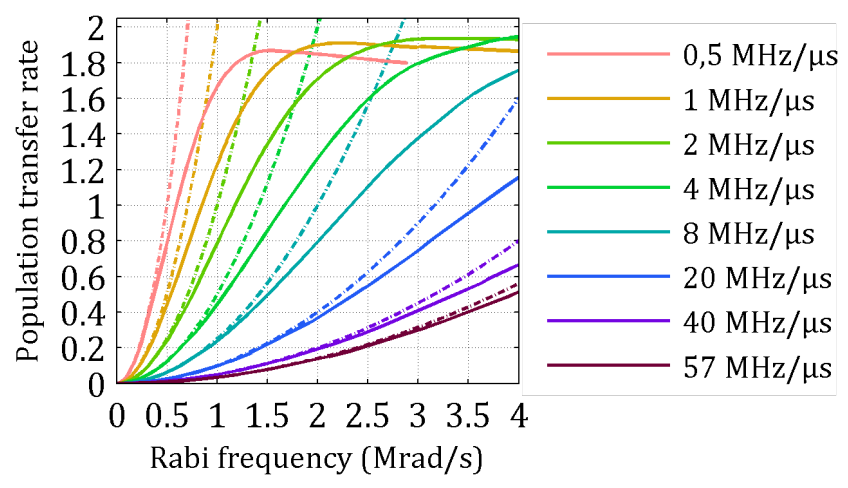

Fig. 7. Simulated population transfer rate on a bright fringe of the spectral interference pattern created by the sum of two chirps defined in Eq. (14), as a function of the Rabi frequency $\Omega_{0}$. Dashed lines correspond to the analytic derivation of the transfer rate using a weak field approximation. The selected chirp rates correspond to $20 \mathrm{GHz}$ swept in $\Delta_{t} \in\{40,20,10,5,2.5,1,0.5,0.35\} \mathrm{ms}$.

For Rabi frequencies up to a few Mrad/s, the computation on our laptop computer (CPU Intel Core i5-3320M @2.6GHz with 4GB RAM) for one set of variables $\left(\Omega_{0}, r\right)$ takes several minutes. This time could be reduced by using lower-level programming. This is not the prospect of the present paper which focuses on the method.

With a compromise between accuracy and computing time, we extend our calculation to larger Rabi frequencies up to $60 \mathrm{Mrad} / \mathrm{s}$. As shown in Fig. 8, the population transfer rate deviates from Eq. (24), and strongly oscillates with the Rabi frequency, similarly to the standard Rabi oscillations. In the double chirp case, considering that a given chirp rate yields an effective pulse duration, the oscillations observed in Fig. 8 can be regarded as "chirped pseudo Rabi oscillations". Indeed, the Bloch vector essentially moves when the third component of the control vector approaches zero, and is approximately tilted by the pulse area (integral of the Rabi frequency $\Omega_{0}$ ).

Considering Eq. (18) and discarding all relaxation processes, we derive an approximated Rabi frequency $\Omega_{0}^{\pi-p u l s e}$ required for a "chirped pseudo $\pi$-pulse" using a geometric argument on the Bloch sphere: $\Omega_{0}^{\pi-p u l s e} \simeq \sqrt{\pi r}$. This expression is reminiscent of the adiabaticity condition of a so-called Rapid Adiabatic Passage with a single chirp, which reads as $\Omega_{0} \gg \sqrt{r}$ [50]. 


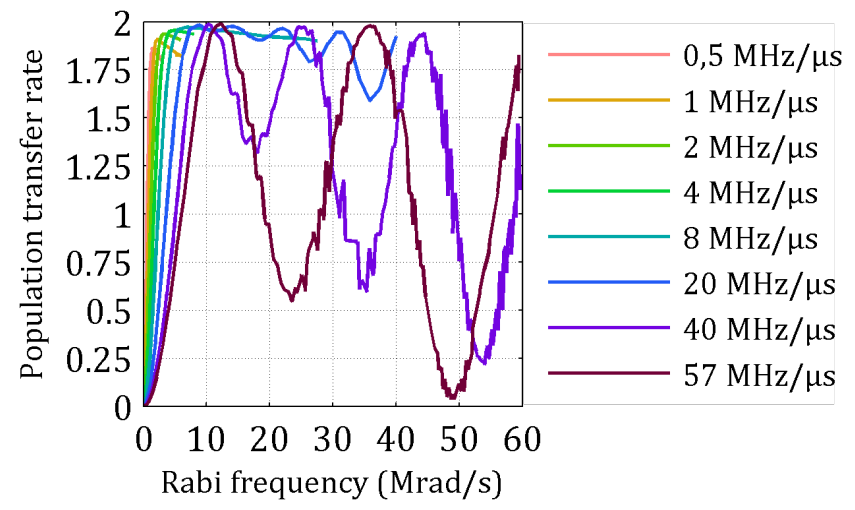

Fig. 8. Simulated population transfer rate as a function of the Rabi frequency for the same chirp rates as in Fig. 7, but much higher Rabi frequencies. The noise comes from the compromise between computation time and accuracy.

This shows the ability of our method to represent coherent lightmatter interaction effects such as adiabatic inversion, in the form of a population transfer rate.

\section{D.2. Propagation in an optically thick medium and diffraction effi- ciency}

With the method described in section C.3, we perform a full calculation of the absorption spectrum within the whole depth of the crystal in a few seconds only, as compared to minutes for the initial tabulation of the transfer rates. An example of the spectral grating through the optical depth is plotted in Fig. 9. The progressive distortion from sinusoidal to square lineshape was predicted in [26] and corresponds to the creation of higher order harmonics arising from the nonlinearity of Eq. (22).

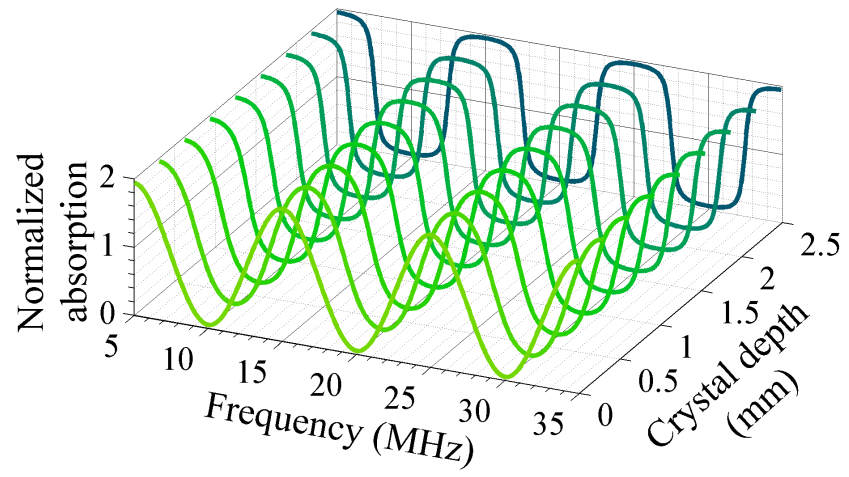

Fig. 9. Simulated spectral gratings obtained for a Rabi frequency $\Omega_{0}=1 \mathrm{Mrad} / \mathrm{s}$ and a chirp rate $r=40 \mathrm{MHz} / \mu \mathrm{s}$ in the depth of the $\mathrm{Tm}^{3+}:$ YAG crystal. The physical depth is $L=2.5 \mathrm{~mm}$ and the optical depth $\alpha_{0} L=2$.

We now apply the method to a broad range of input Rabi frequencies from $10 \mathrm{krad} / \mathrm{s}$ up to $20 \mathrm{Mrad} / \mathrm{s}$. By interpolating the results of Figs. 7 and 8, the full propagation in time and space is achieved for a set of 30 values of the Rabi frequency within less than 10 minutes with our simple laptop computer. The results, averaged over the crystal depth, are plotted in Fig. 10. For weak fields, the grating shape mimics the sinusoidal excitation spectrum as expected from the weak field approximation (see Eq. (8)). The average absorption of the grating is preserved because the population is essentially shuffled between the ground state sublevels. For stronger fields, the population starts accumulating

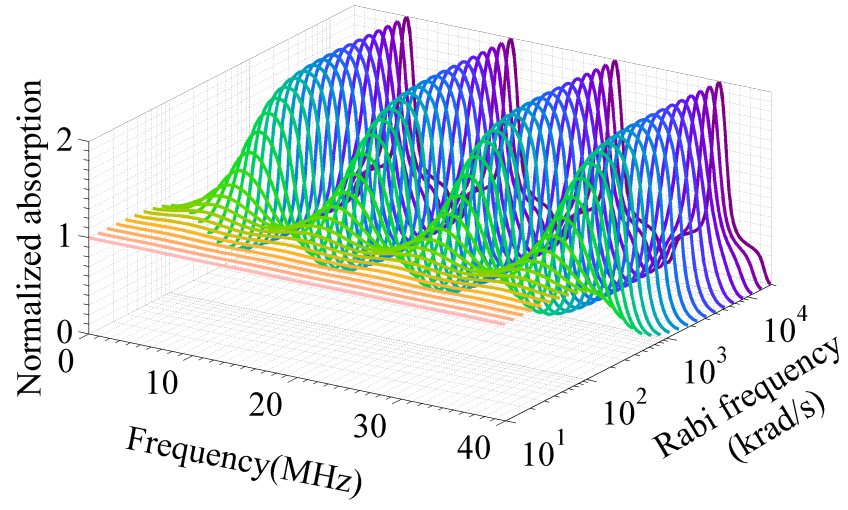

Fig. 10. Simulated spectral gratings obtained for Rabi frequencies ranging from $10 \mathrm{krad} / \mathrm{s}$ to $20 \mathrm{Mrad} / \mathrm{s}$ and for a chirp rate $r=40 \mathrm{MHz} / \mu \mathrm{s}$ in an optically thick medium. The gratings are averaged over the crystal depth.

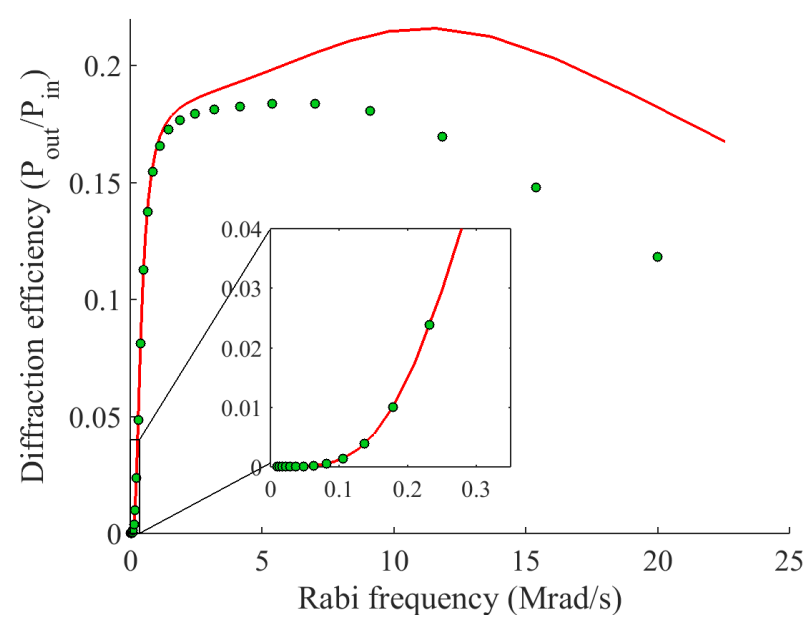

Fig. 11. First order diffraction efficiency calculated from the simulated spectral gratings in Fig. 10 (green dots). As a comparison, we plot the expected efficiency in the weak field approximation where the pumping rate is analytically deduced from the sinusoidal excitation spectrum (see Eq. (8)).

in the metastable state, thus reducing the average absorption. Besides, the grating shape changes, with narrower peaks and holes, because the population transfer rate significantly deviates from the quadratic behavior as a function of the Rabi frequency and even decreases above $10 \mathrm{Mrad} / \mathrm{s}$ (see Fig. 8).

We use the propagation equation Eq. (22) to compute the diffraction efficiency of a probe beam in a given order of diffraction, as explained in [26]. The first order diffraction efficiency is plotted in Fig. 11. For weak fields, the efficiency scales as the fourth power of the Rabi frequency as the contrast of the grating increases and is well predicted by assuming the pumping rate analytically deduced from Eq. (8) (Fig. 11, inset). Deviating from the weak field approximation, the simulated efficiency reaches a maximum (18.3\%) and finally decreases as the features of the grating get narrower. For large efficiencies, the weak field approximation cannot be used, somehow justifying the use of a more complete numerical simulation.

This whole simulation is performed in less than 10 minutes on our laptop computer. Again, faster computing of our method 
could be obtained with lower-level programming. This approach paves the way to the optimization of a broad range of optical signal processing protocols.

\section{CONCLUSION}

We have described a simulation method that predicts the shape of the steady-state absorption profile in an optically thick spectral hole-burning material under periodic excitation. The lightatom interaction during a single excitation pattern is numerically solved and rewritten as a transfer matrix, with an equivalent population transfer rate. This approach accounts for coherent excitation and relaxation. The weak field approximation which strongly simplifies the theoretical treatment cannot be used in the parameters range where the largest efficiencies are expected.

In our case, we use the Bloch vector formalism in order to deal with real variables to minimize the computational resources. The steady-state atomic populations are simply expressed as a matrix eigenvector. Under the assumption of a modest field distortion during propagation, this numerical resolution is performed only once, even when dealing with an optically thick medium. This way the time evolution and the propagation through the medium are decoupled.

This method is practical for any signal processing architectures based on accumulated programming of a spectral structure in the absorption profile, including spectral analysis of opticallycarried RF signals, ultrasound-optical tomography, and quantum memories. It can be directly adapted to atomic vapors studied by femtosecond pulses, thus finding applications in high resolution spectroscopy and metrology with frequency-combs. The approach can be extended to a large number of levels or optical transitions involved in the optical pumping process. Because we track the population changes after each excitation step, detrimental effects such as spectral diffusion could be included by shuffling the populations between the steps. Our method can also account for a spatial field dependency in the transverse $(x, y)$ plane as Gaussian beams. The only geometric limitations would come from an imperfect overlap of several beams.

\section{ACKNOWLEDGMENTS}

We thank Jean-Louis Le Gouët for helpful discussions. This work has been realized in the framework of the joint research laboratory between Laboratoire Aimé Cotton and Thales Research \& Technology. This work was partly funded by the Direction Générale de l'Armement (DGA).

\section{A. GENERALIZED OPTICAL BLOCH EQUATIONS}

Let us consider a generalized atomic structure $\mathcal{S}_{A}$ composed of $N_{o}$ optical transitions and $N_{L}$ atomic levels. We denote the states $|\psi\rangle_{i}, i \in \llbracket 1, N_{L} \rrbracket$ and $n_{i}$ the population in state $|i\rangle$.

Unlike previous works on the general Bloch vector that aimed at formalizing the multi-dimensional "Bloch-ball" and identifying its mathematical properties [45], we aim at deriving the most practical formulation of the vector Bloch equations in such a system, similar to Eq. (10).

We consider the total field that can arise from the addition of several fields $\overrightarrow{\mathcal{E}}(t, \vec{r})=\sum_{q}\left(\vec{E}_{0, q}(t) e^{i \phi_{q}(t, \vec{r})}+\right.$ c.c. $)$. For instance, using these notations, a simple monochromatic square pulse of duration $T$ and optical frequency $v_{0}$ reads as $\vec{E}_{0}$. $\mathbb{1}_{[0, T]}(t) \cdot e^{-2 i \pi v_{0} t+i \vec{k} \cdot \vec{r}}+$ c.c.. For every optical transition between state $|a\rangle$ and state $|b\rangle$, we can define the Rabi frequency
$\Omega_{a b}(t, \vec{r})=\vec{\mu}_{a b} \cdot \overrightarrow{\mathcal{E}}(t, \vec{r}) / \hbar$, where $\vec{\mu}_{a b}$ is the electric dipole of the transition. The complex phase of $\Omega_{a b}$ can be split into a fast part $\phi_{a b}^{(F)}(t, \vec{r})$ and a slow part $\phi_{a b}^{(S)}(t, \vec{r})$, where $\phi_{a b}^{(F)}(t, \vec{r})$ varies in the time domain with the frequency of the optical wave $v_{0}$ and in the space domain with the wavevector $k$ along the $\hat{z}$ direction defined in Sec. D. Finally, the Rabi frequency can be decomposed in the following way:

$$
\Omega_{a b}(t, \vec{r})=\Omega_{a b}^{(M)} \cdot e^{i\left(\phi_{a b}^{(S)}+\phi_{a b}^{(F)}\right)}=\Omega_{a b}^{(C)} \cdot e^{i \phi_{a b}^{(F)}},
$$

where $\Omega_{a b}^{(C)}$ is the complex, slowly varying part of the Rabi frequency. We define the variable changes enabling the use of the rotating wave approximation [44]:

$$
\begin{aligned}
\sigma_{a a} & =\rho_{a a}=n_{a}, \\
\sigma_{b b} & =\rho_{b b}=n_{b}, \\
\sigma_{a b} & =\rho_{a b} e^{i \phi_{a b}^{(F)}}, \\
\sigma_{b a} & =\rho_{b a} e^{-i \phi_{a b}^{(F)}} .
\end{aligned}
$$

The control vector $\vec{\beta}_{a b}$ reads as:

$$
\vec{\beta}_{a b}=\left(\begin{array}{c}
\Re\left(\Omega_{a b}^{(C)}\right) \\
\Im\left(\Omega_{a b}^{(C)}\right) \\
-\Delta_{a b}
\end{array}\right),
$$

where $\Delta_{a b}$ is the detuning between the atomic resonance and the mean frequency of the electric field. For each couple of states $|a\rangle$ and $|b\rangle$ linked by an optical transition, we define a Bloch vector $\left(U_{a b} ; V_{a b} ; W_{a b}\right)$ :

$$
\begin{aligned}
U_{a b} & =\sigma_{a b}+\sigma_{b a}, \\
V_{a b} & =i\left(\sigma_{a b}-\sigma_{b a}\right), \\
W_{a b} & =n_{b}-n_{a} .
\end{aligned}
$$

To fully solve the time evolution of the atomic state, one needs to determine the complete set of variables to include in the formalism. We first consider all the $N_{o}$ Bloch vectors corresponding to the optical transitions and gather them into a vector $\vec{B}_{o}$ (of size $3 N_{o}$ ). In order to count the number of independent elements of this vector, we must identify cycles of transitions. We define a cycle of transitions as an ensemble of 2 or more transitions $\left\{\left(i_{1}, i_{2}\right),\left(i_{2}, i_{3}\right), \ldots,\left(i_{p-1}, i_{p}\right),\left(i_{p}, i_{1}\right)\right\}$. For every cycle, since the variable $W_{p, 1}$ can be calculated from all the other $W_{i, j}$ of the cycle, it can be withdrawn from vector $\vec{B}_{0}$. The size of $\vec{B}_{o}$ is then $3 N_{o}-N_{\text {cycle }}$, where $N_{\text {cycle }}$ is the number of cycles of transitions. Note that every time a cycle is "broken" (ie a variable of type $W_{p, 1}$ is withdrawn), one has to redefine the remaining cycles since several cycles may be entangled and simultaneously broken. Once $\vec{B}_{o}$ is clear of cycles, for every set of contiguous states (linked by optical transitions), one can choose one state of the set and define a variable $X_{i}=n_{i}$ that is simply the atomic population in state $|i\rangle$. If possible, the chosen state should be on the edge of the set so that it involves only one optical transition. All these $X_{i}$ constitute a population vector $\vec{B}_{P_{1}}$ (of size $N_{P}$ ). Since the structure has $N_{L}$ atomic levels, $N_{L}$ population variables are required. Yet, $N_{o}-N_{\text {cycle }}+N_{P}$ population variables are already defined. Therefore, $N_{L}-\left(N_{o}-N_{\text {cycle }}+N_{P}\right.$ other variables of type $Y_{i}=n_{i}$ chosen among the other states only reached by relaxation processes must be considered. These variables can 
be gathered into a vector $\vec{B}_{P_{2}}=\left(Y_{1} ; Y_{2} ; \ldots ; Y_{N_{L}-\left(N_{O}-N_{\text {cycle }}+N_{P}\right)}\right)$. The full generalized Bloch vector reads as:

$$
\vec{B}=\left(\vec{B}_{0} ; \vec{B}_{P_{1}} ; \vec{B}_{P_{2}}\right) \text {. }
$$

The relaxation vector $\vec{R}$ defined in Eq. (10) and acting on $\vec{B}$ can finally be spelled.

Now one can write the full set of Bloch equations as a pure vector equation, similar to equation Eq. (10):

$$
\partial_{t}\left(\begin{array}{c}
\vec{B}_{o} \\
\vec{B}_{P_{1}} \\
\vec{B}_{P_{2}}
\end{array}\right)=\left(\begin{array}{c}
\vec{P}_{o} \\
\vec{P}_{P_{1}} \\
\vec{P}_{P_{2}}
\end{array}\right)+\vec{R}=\vec{P}+\vec{R} .
$$

In Eq. (35), $\vec{P}_{o}$ is composed of triplets of components that read as:

$$
\begin{array}{r}
\overrightarrow{\beta_{a b}} \times\left(\begin{array}{c}
U_{a b} \\
V_{a b} \\
W_{a b}
\end{array}\right) \\
+\frac{1}{2} \sum_{p \in \mathcal{N}(a), p \neq b}\left(\left(\begin{array}{c}
\vec{\beta}_{a p}^{(1)} \\
\vec{\beta}_{a p}^{(2)} \\
0
\end{array}\right) \times\left(\begin{array}{c}
U_{a p} \\
V_{a p} \\
0
\end{array}\right)\right) \\
-\frac{1}{2} \sum_{q \in \mathcal{N}(b), q \neq a}\left(\left(\begin{array}{c}
\vec{\beta}_{b q}^{(1)} \\
\vec{\beta}_{b q}^{(2)} \\
0
\end{array}\right) \times\left(\begin{array}{c}
U_{b q} \\
V_{b q} \\
0
\end{array}\right)\right),
\end{array}
$$

where $\mathcal{N}(i)$ stands for the ensemble of all the neighboring atomic states of $|i\rangle$ (linked by an optical transition). The exponents (1) and ${ }^{(2)}$ stand for the first and the second components of $\vec{\beta}_{i j}$ vectors. In the case of non-contiguous optical transitions (as in Sec. 4 of this article), the two sums disappear. Finally in Eq. (35), $\vec{P}_{P_{1}}$ reads as:

$$
\vec{P}_{P_{1}}=\left(\begin{array}{c}
\sum_{p \in N(1)}\left(\vec{\beta}_{1 p}^{(1)} V_{1 p}-\vec{\beta}_{1 p}^{(2)} U_{1 p}\right) \\
\vdots \\
\sum_{p \in \mathcal{N}\left(N_{p}\right)}\left(\vec{\beta}_{N_{p} p}^{(1)} V_{N_{p} p}-\vec{\beta}_{N_{p} p}^{(2)} U_{N_{p} p}\right)
\end{array}\right)
$$

and $\vec{P}_{P_{2}}=\overrightarrow{0}$. The components of vector $\vec{P}_{P_{1}}$ account for the population change in terms of $X_{i}$ variables. These come from the cross products that appear in Eq. (36).

Using the expressions of all the components of the Bloch vector $\vec{B}$, the pumping vector $\vec{P}$, and the relaxation vector $\vec{R}$, one can numerically solve the Bloch equation given in Eq. (35) and get the time evolution of the system. Such a generalized expression of the light matter interaction can then be utilized to derive the population transfer rates $\Pi_{a b}$ and yield the steady state absorption profile of a multilevel system under periodic excitation.

\section{REFERENCES}

1. A. K. Rebane, R. K. Kaarli, and P. M. Saari, "Hole-burning by coherent sequencies of picosecond pulses," Opt. Spectrosk. 55, 405-407 (1983).

2. A. Rebane, R. Kaarli, P. Saari, A. Anijalg, and K. Timpmann, "Photochemical time-domain holography of weak picosecond pulses," Opt. Commun. 47, 173-176 (1983).
3. P. Saari, R. Kaarli, and A. Rebane, "Picosecond time- and spacedomain holography by photochemical hole burning," J. Opt. Soc. Am. B 3, 527-534 (1986).

4. A. Rebane, "Associative space-and-time domain recall of picosecond light signals via photochemical hole burning holography," Opt. communications 65, 175-178 (1988).

5. T. W. Mossberg, "Time-domain frequency-selective optical data storage," Opt. Lett. 7, 77-79 (1982).

6. Y. Bai, W. Babbitt, and T. W. Mossberg, "Coherent transient optical pulse-shape storage/recall using frequency-swept excitation pulses," Opt. letters 11, 724-726 (1986).

7. T. W. Mossberg, "Swept-carrier time-domain optical memory," Opt. Lett. 17, 535-537 (1992).

8. W. Babbitt and T. Mossberg, "Time-domain frequency-selective optical data storage in a solid-state material," Opt. communications 65, 185188 (1988).

9. M. Mitsunaga and N. Uesugi, "248-bit optical data storage in eu 3+: Yalo 3 by accumulated photon echoes," Opt. letters 15, 195-197 (1990).

10. M. Mitsunaga, R. Yano, and N. Uesugi, "Time-and frequency-domain hybrid optical memory: 1.6-kbit data storage in eu 3+: Y 2 sio 5," Opt. letters 16, 1890-1892 (1991).

11. K. Merkel, R. K. Mohan, Z. Cole, T. Chang, A. Olson, and W. Babbitt, "Multi-gigahertz radar range processing of baseband and if carrier modulated signals in tm: Yag," J. Lumin. 107, 62-74 (2004).

12. G. Gorju, V. Crozatier, I. Lorgeré, J.-L. Le Gouët, and F. Bretenaker, "10-ghz bandwidth rf spectral analyzer with mhz resolution based on spectral hole burning in tm/sup 3+: Yag," IEEE Photonics Technol. Lett. 17, 2385-2387 (2005).

13. K. Heshami, D. G. England, P. C. Humphreys, P. J. Bustard, V. M. Acosta, J. Nunn, and B. J. Sussman, "Quantum memories: emerging applications and recent advances," J. modern optics 63, 2005-2028 (2016).

14. M. Afzelius, C. Simon, H. de Riedmatten, and N. Gisin, "Multimode quantum memory based on atomic frequency combs," Phys. Rev. A 79, 052329 (2009).

15. V. Crozatier, V. Lavielle, F. Bretenaker, J.-L. Le Gouët, and I. Lorgeré, "High-resolution radio frequency spectral analysis with photon echo chirp transform in an Er: YSO crystal," Quantum Electron. IEEE J. 40, 1450-1457 (2004).

16. H. Linget, L. Morvan, J.-L. Le Gouët, and A. Louchet-Chauvet, "Time reversal of optically carried radiofrequency signals in the microsecond range," Opt. Lett. 38, 643-645 (2013).

17. E. Saglamyurek, N. Sinclair, J. A. Slater, K. Heshami, D. Oblak, and W. Tittel, "An integrated processor for photonic quantum states using a broadband light-matter interface," New J. Phys. 16, 065019 (2014).

18. I. Lorgeré, L. Ménager, V. Lavielle, J.-L. Le Gouët, D. Dolfi, S. Tonda, and J.-P. Huignard, "Demonstration of a radio-frequency spectrum analyser based on spectral hole burning," J. Mod. Opt. 49, 2459-2475 (2002).

19. K. D. Merkel and W. R. Babbitt, "Chirped-pulse programming of optical coherent transient true-time delays," Opt. Lett. 23, 528 (1998).

20. Y. Li, P. Hemmer, C. Kim, H. Zhang, and L. Wang, "Detection of ultrasound-modulated diffuse photons using spectral-hole burning." Opt. Exp. 16, 14862 (2008).

21. K. Kutluer, M. F. Pascual-Winter, J. Dajczgewand, P. M. Ledingham, M. Mazzera, T. Chanelière, and H. de Riedmatten, "Spectral-hole memory for light at the single-photon level," Phys. Rev. A 93, 040302 (2016).

22. S. Cook, T. Rosenband, and D. R. Leibrandt, "Laser-frequency stabilization based on steady-state spectral-hole burning in $\mathrm{Eu}^{3+}: \mathrm{Y}_{2} \mathrm{SiO}_{5}$," Phys. Rev. Lett. 114, 253902 (2015).

23. J. W. Tay, W. G. Farr, P. M. Ledingham, D. Korystov, and J. J. Longdell, "Hybrid optical and electronic laser locking using slow light due to spectral holes," Phys. Rev. A 87, 063824 (2013).

24. W. H. Hesselink and D. A. Wiersma, "Picosecond photon echoes stimulated from an accumulated grating," Phys. Rev. Lett. 43, 1991 (1979).

25. M. Bonarota, J.-L. L. Gouët, and T. Chanelière, "Highly multimode 
storage in a crystal," New J. Phys. 13, 013013 (2011)

26. H. Linget, T. Chanelière, J.-L. Le Gouët, P. Berger, L. Morvan, and A. Louchet-Chauvet, "Interlaced spin gratings for optical wave filtering," Phys. Rev. A 91, 023804 (2015).

27. W. H. Hesselink and D. A. Wiersma, "Photon echoes stimulated from an accumulated grating: theory of generation and detection," The J. Chem. Phys. 75, 4192-4197 (1981).

28. O. K. Khasanov, T. Smirnov, and O. Fedotova, "Multipulse generation of stimulated photon echo signals in optically dense media," Opt. Spectrosc. 82, 260-263 (1997).

29. K. Merkel, R. Peters, P. Sellin, K. Repasky, and W. Babbitt, "Accumulated programming of a complex spectral grating," Opt. Lett. 25, 1627-1629 (2000).

30. M. Tian, J. Zhao, Z. Cole, R. Reibel, and W. R. Babbitt, "Dynamics of broadband accumulated spectral gratings in $\mathrm{Tm}^{3+}:$ YAG," J. Opt. Soc. Am. B 18, 673-678 (2001).

31. H. de Vries and D. A. Wiersma, "Numerical simulations of accumulated stimulated photon echoes," J. Chem. Phys. 80, 657-666 (1984).

32. D. Felinto, L. Acioli, and S. Vianna, "Accumulative effects in the coherence of three-level atoms excited by femtosecond-laser frequency combs," Phys. Rev. A 70, 043403 (2004).

33. W. Yang, S. Gong, R. Li, and Z. Xu, "Coherent population accumulations of multiphoton transitions induced by an ultrashort pulse train in polar molecules," Phys. Rev. A 74, 013407 (2006).

34. T. Ban, D. Aumiler, H. Skenderović, and G. Pichler, "Mapping of the optical frequency comb to the atom-velocity comb," Phys. Rev. A 73, 043407 (2006).

35. T. Ban, D. Aumiler, H. Skenderović, S. Vdović, N. Vujičić, and G. Pichler, "Cancellation of the coherent accumulation in rubidium atoms excited by a train of femtosecond pulses," Phys. Rev. A 76, 043410 (2007).

36. H. Tang and T. Nakajima, "Effects of the pulse area and pulse number on the population dynamics of atoms interacting with a train of ultrashort pulses," Opt. Commun. 281, 4671-4675 (2008).

37. D. Aumiler, T. Ban, and G. Pichler, "Time dynamics of a multilevel system excited by a train of ultrashort pulses," Phys. Rev. A 79, 063403 (2009).

38. T. Ban, D. Aumiler, S. Vdović, N. Vujičić, H. Skenderović, and G. Pichler, "Coherent population dynamics in rubidium atoms excited by resonant $0 \pi$ pulses," Phys. Rev. A 80, 023425 (2009).

39. N. Vujičić, T. Ban, G. Kregar, D. Aumiler, and G. Pichler, "Velocityselective double resonance in doppler-broadened rubidium vapor," Phys. Rev. A 87, 013438 (2013).

40. S. T. Cundiff and S. Mukamel, "Optical multidimensional coherent spectroscopy," Phys. Today 66, 44 (2013).

41. R. W. Boyd, Nonlinear optics (Academic press, 2003).

42. P. W. Milonni, Fast light, slow light and left-handed light (CRC Press, 2004).

43. J. S. Toll, "Causality and the dispersion relation: logical foundations," Phys. Rev. 104, 1760 (1956).

44. L. Allen and J. H. Eberly, Optical Resonance and Two-Level Atoms (Courier Dover Publications, 1987).

45. G. Kimura, "The Bloch vector for N-level systems," Phys. Lett. A 314, 339-349 (2003).

46. P. Berger, M. Schwarz, S. Molin, D. Dolfi, L. Morvan, A. LouchetChauvet, T. Chanelière, and J.-L. Le Gouët, " $20 \mathrm{GHz}$ instantaneous bandwidth RF spectrum analyzer with high time-resolution," in "Microwave Photonics (MWP) and the 2014 9th Asia-Pacific Microwave Photonics Conference (APMP), 2014 International Topical Meeting on," (IEEE, 2014), pp. 331-334.

47. F. de Seze, A. Louchet, V. Crozatier, I. Lorgeré, F. Bretenaker, J.-L. Le Gouët, O. Guillot-Noël, and P. Goldner, "Experimental tailoring of a three-level $\Lambda$ system in $\mathrm{Tm}^{3+}$ :YAG," Phys. Rev. B 73, 085112 (2006).

48. Y. Sun, G. Wang, R. Cone, R. Equall, and M. Leask, "Symmetry considerations regarding light propagation and light polarization for coherent interactions with ions in crystals," Phys. Rev. B 62, 15443 (2000).

49. L. F. Shampine and M. W. Reichelt, "The matlab ode suite," SIAM J. on Sci. Comput. 18, 1-22 (1997).
50. N. V. Vitanov, T. Halfmann, B. W. Shore, and K. Bergmann, "Laserinduced population transfer by adiabatic passage techniques," Annu. Rev. Phys. Chem. 52, 763 (2001). 